\title{
PRODUTIVIDADE E FITODISPONIBILIDADE DE NUTRIENTES NA TIFTON 85 (Cynodon dactylon) FERTILIZADA COM DEJETOS PROVENIENTES DA SUINOCULTURA
}

\section{PRODUCTIVITY AND BIOAVAILABILITY OF NUTRIENTS IN TIFTON 85 (Cynodon dactylon) FERTILIZED WITH WASTE FROM HOGS}

\author{
Ricardo ZENATTI ${ }^{1}$ \\ Affonso Celso GONÇALVES JR ${ }^{2}$ \\ Herbert NACKE ${ }^{3}$ \\ Ivan RAMIRES ${ }^{4}$
}

\section{RESUMO}

Foi avaliado o efeito da aplicação de doses crescentes de dejetos de suínos (in natura e biofertilizante) na produção e no teor de nutrientes na forrageira cv. Tifton 85 (Cynodon dactylon). O experimento foi implantado em ambiente protegido, em um solo classificado como Argissolo Vermelho distrófico ( $P V d)$. Os tratamentos foram constituídos por duas fontes de fertilizantes orgânicos (in natura e biofertilizante), com quatro doses para cada fertilizante orgânico e quatro repetições. Os resultados apresentaram efeito significativo entre as fontes e as doses, sendo que a produção média da gramínea no primeiro corte com base na massa fresca foi de $9953 \mathrm{~kg} \mathrm{ha}^{-1}$ e para massa seca $4445 \mathrm{~kg} \mathrm{ha}^{-1}$, as fontes influenciaram nos teores de $P$ e Mn, e as doses influenciaram nos teores de N, P, K, Cu e Zn. No segundo corte a média para massa fresca foi de $8711 \mathrm{~kg}$ $\mathrm{ha}^{-1}$ e para massa seca $3782 \mathrm{~kg} \mathrm{ha}^{-1}$, as fontes influenciaram nos teores dos elementos $\mathrm{P}, \mathrm{Fe}, \mathrm{Mn}$, e $\mathrm{Cu}$ e as doses influenciaram nos teores de N, P, K, Fe, Mn, Cu. Conclui-se que a aplicação de dejetos in natura proporcionou maior produção da cv Tifton 85 quando comparada com uso de biofertilizante, conclui-se ainda que os teores foliares dos nutrientes $\mathrm{N}, \mathrm{P}$ e K e dos micronutrientes $\mathrm{Fe}$, Cu e $\mathrm{Zn}$ aumentaram em função das doses aplicadas e das fontes utilizadas e o elemento Mn diminuiu em função das doses aplicadas.

Palavras chave: suínos, fertilização orgânica, gramínea.

\begin{abstract}
The aim of this work was evaluate the application of increasing doses of swine manure (in nature and biofertilizer) and their effect in production and nutrient content in grass (Cynodon dactylon). The experiment was carried out in greenhouse, in a soil classified as Typic Rhodustults. The treatments was consisted by two fertilizer organic sources (in nature and biofertilizer), with four doses for each source and four repetitions. The results showed significant effect among the sources and doses, being that the average production of the grass in the first cut based on fresh weight was $9953 \mathrm{~kg} \mathrm{ha}^{-1}$ and for dry weight 4445 $\mathrm{kg} \mathrm{ha}^{-1}$, the sources influenced content of $\mathrm{P}$ and $\mathrm{Mn}$, and the doses influenced contents of $\mathrm{N}, \mathrm{P}, \mathrm{K}, \mathrm{Cu}$ and $\mathrm{Zn}$. In the second cut to the average fresh weight was $8711 \mathrm{~kg} \mathrm{ha}^{-1}$ and dry weight $3782 \mathrm{~kg} \mathrm{ha}^{-1}$, the sources influenced the contents of the elements $\mathrm{P}, \mathrm{Fe}, \mathrm{Mn}$, and $\mathrm{Cu}$ and the doses influenced the content of $\mathrm{N}, \mathrm{P}, \mathrm{K}, \mathrm{Fe}, \mathrm{Mn}, \mathrm{Cu}$. It was concluded that the application of manure in nature provided bigger production of cv Tifton 85 compared with the use of biofertilizer, it also concludes that the leaf contents of $\mathrm{N}, \mathrm{P}$ and $\mathrm{K}$ and the micronutrients $\mathrm{Fe}, \mathrm{Cu}$ and $\mathrm{Zn}$ was increased in function of the doses and sources used and the element Mn decreased in function of the applied doses.
\end{abstract}

Key-words: swine, organic fertilization, grass.

\footnotetext{
${ }^{1}$ Estudante, Programa de Pós-Graduação em Agronomia. Universidade Estadual do Oeste do Paraná, Rua Pernambuco, 1777, Marechal Cândido Rondon, PR, Brasil, 85960 000. E-mail: ricardoze83@hotmail.com.

2 Centro de Ciências Agrárias, Universidade Estadual do Oeste do Paraná. Rua Pernambuco, 1777, Marechal Cândido Rondon, PR, Brasil, 85960000.

${ }^{3}$ Estudante, Programa de Pós-Graduação em Agronomia. Universidade Estadual do Oeste do Paraná, Rua Pernambuco, 1777, Marechal Cândido Rondon, PR, Brasil, 85960 000. E-mail: hnacke@hotmail.com.

${ }^{4}$ Departamento de Química, Universidade Federal da Grande Dourados, MS. E-mail: inobj@hotmail.com
} 
ZENATTI , R. et al. Produtividade e fitodisponibilidade...

\section{INTRODUÇÃO}

A utilização de gramíneas de alta produtividade, para a manutenção da cobertura do solo em regiões tropicais tem sido uma alternativa para elevar a produção das pastagens (Vilela et al., 1996). Neste sentido, o capim"Tifton 85" (Cynodon dactylon) apresenta-se como uma alternativa promissora devido a suas excelentes características produtivas, sendo grande o número de produtores que têm utilizado essa forrageira (Sarmiento, 2006).

Para a escolha de uma determinada cultivar de alta produtividade, é necessário suprir as necessidades de nutrientes da cultura, e uma prática crescente, conforme Durigon et al. (2002), é a utilização de dejetos suínos na fertilização dos pastos. Entretanto, a quantidade a ser aplicada é de suma importância no contexto da viabilidade econômica e sustentabilidade do processo de utilização dos dejetos para que não se inicie um problema ainda maior, que é a degradação ambiental (Gonçalves Jr. et al., 2008).

As doses a serem aplicadas no solo irão depender do potencial fertilizante, do resultado de análise do solo e das exigências da cultura (Miranda et al., 1999). Não obstante, de acordo com Queiroz et al. (2004), para um aproveitamento adequado dos dejetos, é fundamental estabelecer uma dosagem de aplicação, evitando o desequilíbrio de nutrientes.
Outro importante fator a ser levado em consideração é a forma do dejeto a ser utilizado, sendo que estes podem ser aplicados sem tratamento (in natura) e/ou fermentado anaerobicamente (biofertilizante), podendo assim, interferir na disponibilidade de nutrientes, produção da forrageira e no impacto ao ambiente.

Nesse sentido o objetivou-se avaliar os efeitos de doses crescentes de dejetos in natura e biofertilizante de suínos na produção e nos teores de nutrientes da gramínea cv. Tifton 85 (Cynodon dactylon).

\section{MATERIAL E MÉTODOS}

O experimento foi conduzido na casa de vegetação da Universidade Estadual do Oeste do Paraná no município de Marechal Cândido Rondon-PR que possui as seguintes coordenadas geográficas: $24^{\circ} 31^{\prime} \mathrm{S}$ e $54^{\circ} 01^{\prime} \mathrm{W}$. Para tanto utilizou-se vasos contendo $8 \mathrm{dm}^{3}$ de um solo classificado como Argissolo Vermelho distrófico (PVd) (Empresa Brasileira de Pesquisa Agropecuária, 2006), apresentando textura arenosa, $\left(218,50 \mathrm{~g} \mathrm{~kg}^{-1}\right.$ de argila, $69,28 \mathrm{~g} \mathrm{~kg}^{-}$ ${ }^{1}$ de silte e $712,22 \mathrm{~g} \mathrm{~kg}^{-1}$ de areia).

$\mathrm{Na}$ Tabela 1 são apresentados os resultados da análise química do solo antes da instalação do experimento. Como metodologia foi utilizada o manual de análises químicas de solo do Instituto Agronômico do Paraná (Pavan et al., 1992).

TABELA 1 - Análise química do Argissolo Vermelho distrófico (PVd) utilizado no experimento

\begin{tabular}{|c|c|c|c|c|c|c|c|c|c|c|c|c|c|}
\hline \multirow{2}{*}{$\begin{array}{c}\mathrm{pH} \\
\left(\mathrm{CaCl}_{2}\right)\end{array}$} & $\mathrm{K}$ & $\mathrm{Ca}$ & $\mathrm{Mg}$ & $\mathrm{H}+\mathrm{Al}$ & SB & СтС & C & $P$ & $\mathrm{Cu}$ & $\mathrm{Zn}$ & $\mathrm{Fe}$ & $\mathrm{Mn}$ & V\% \\
\hline & --- & -..-. & $-c r$ & ${ }_{c} \mathrm{dm}$ & --.-- & -- & $\mathrm{g} \mathrm{dm}^{-3}$ & ----. & ------ r & $\lg \mathrm{dr}$ & 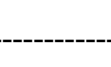 & & $\%$ \\
\hline 5,18 & 0,85 & 5,16 & 1,53 & 5,79 & 7,50 & 13,30 & 25,90 & $\begin{array}{c}18,7 \\
0\end{array}$ & 11,20 & $\begin{array}{c}282,0 \\
0\end{array}$ & 33,40 & 5,20 & 56,50 \\
\hline
\end{tabular}

$\mathrm{H}+\mathrm{Al}$ (acidez potencial), SB (soma de bases), CTC (capacidade de troca catiônica), C (carbono orgânico), V\% (saturação por bases), $\mathrm{Cu}, \mathrm{Zn}, \mathrm{Fe}$ e Mn extraídos por Mehlich-1.

O delineamento experimental utilizado neste trabalho foi o inteiramente casualizado, em um arranjo fatorial, sendo os tratamentos constituídos por duas fontes de fertilizantes orgânicos (dejeto in natura e biofertilizante), com quatro doses para cada fonte $\left(0,200,400\right.$ e $\left.600 \mathrm{~m}^{3} \mathrm{ha}^{-1}\right)$ e quatro repetições, totalizando 32 parcelas experimentais.

Para recomendação de calagem e fertilização utilizou-se a Circular Técnica 128 do Instituto Agronômico do Paraná (2003), onde se recomenda a elevação de saturação de bases para a cv. Tifton em $70 \%$, assim foram aplicados 2,94 ton $\mathrm{ha}^{-1}(1,47 \mathrm{~g}$ vaso $\left.^{-1}\right)$. Para a recomendação de fertilização de implantação da cultura, aplicou-se $30 \mathrm{~kg} \mathrm{ha}^{-1}$ de $\mathrm{P}_{2} \mathrm{O}_{5}$ e de $60 \mathrm{~kg} \mathrm{ha}^{-1} \mathrm{~K}_{2} \mathrm{O}$, assim o formulado $\mathrm{N}: \mathrm{P}_{2} \mathrm{O}_{5}: \mathrm{K}_{2} \mathrm{O}$ aplicado teve a proporção $0: 20: 20$, sendo que todos tratamentos receberam a mesma quantidade de fertilizantes químicos antes da im- plantação da cultura.

O plantio das mudas foi realizado em 0506 2010 nos vasos plásticos com capacidade de $8 \mathrm{~L}$, sendo cinco mudas por vaso. Ao atingirem a altura de $20 \mathrm{~cm}$ foi realizado o corte de nivelamento da gramínea a $5 \mathrm{~cm}$ do solo.

$O$ dejeto in natura e o biofertilizante foram coletados em diferentes granjas, os dejetos foram coletados em sistema sem o tratamento (in natura), e o biofertilizante foi coletado após a passagem pelo biodigestor, localizadas no município de Marechal Cândido Rondon-PR, sendo que os fertilizantes foram recolhidos por bombeamento das esterqueiras, e colocados em galões de $50 \mathrm{~L}$. Antes da utilização dos fertilizantes orgânicos foram retiradas alíquotas para sua caracterização química (Tabela 2). 
ZENATTI , R. et al. Produtividade e fitodisponibilidade...

TABELA 2 - Caracterização química do dejeto in natura e do biofertilizante utilizados no experimento

\begin{tabular}{cccccccccc}
\hline \multirow{2}{*}{ Fontes } & N & P & $K$ & Ca & Mg & Mn & Cu & Zn & Fe \\
Dejeto in natura & 15,75 & 16,26 & 2,40 & 5,20 & 1,45 & 111,00 & 40,00 & 85,00 & 38,70 \\
Biofertilizante & 10,51 & 12,30 & 1,80 & 3,49 & 1,00 & 20,00 & 109,00 & 10,50 & 120,00 \\
\hline
\end{tabular}

Para determinação dos elementos fósforo $(\mathrm{P})$, potássio $(\mathrm{K})$, cálcio $(\mathrm{Ca})$, magnésio $(\mathrm{Mg})$, Ferro $(\mathrm{Fe})$, manganês $(\mathrm{Mn})$, cobre $(\mathrm{Cu})$ e zinco $(\mathrm{Zn})$ realizou-se digestão nitro-perclórica (Association of Official Analytical Chemists, 2005), sendo a determinação do $\mathrm{P}$ realizada por espectroscopia de ultra violeta visível (UV-VIS) e os demais nutrientes determinados por espectrometria de absorção atômica, modalidade chama (EAA-Chama). (Welz e Sperling, 1999).

Para determinação dos teores de nitrogênio (N) nos fertilizantes orgânicos foi utilizada a digestão sulfúrica (Association of Official Analytical Chemists, 2005), com determinação por destilação Kjeldahl.

As doses avaliadas foram parceladas em oito aplicações, realizada a cada sete dias. Durante o período do experimento as plantas foram irrigadas a cada dois dias, sendo o volume de água controlado até o momento da saturação do solo.

Para avaliação da produtividade e fitodisponibilidade dos nutrientes foram realizados dois cortes na gramínea cv. Tifton 85. Assim realizou-se o primeiro e o segundo corte, respectivamente, aos 28 e 56 dias após o corte de nivelamento (Santos, 2006).

Para determinação da massa fresca, todo o material vegetal coletado no corte foi pesado em balança analítica. Posteriormente as amostras foram desidratadas em estufa a $45^{\circ} \mathrm{C}$, até atingirem massa constante, sendo novamente pesadas, obtendo-se a massa seca (Drumond et al., 2006).
Para determinação dos elementos $\mathrm{N}, \mathrm{P}, \mathrm{K}$, $\mathrm{Ca}, \mathrm{Mg}, \mathrm{Fe}, \mathrm{Mn}, \mathrm{Cu}$ e $\mathrm{Zn}$ no material vegetal de ambos os cortes foi utilizada a mesma metodologia descrita anteriormente para caracterização dos fertilizantes orgânicos.

Para análise estatística dos dados obtidos no experimento utilizou-se o software SISVAR (Ferreira, 2003). Os dados foram submetidos à análise de variância na significância de 1 e 5\%, em caso de significância entre as fontes foi realizado o teste Tukey a $5 \%$ de probabilidade e para a s doses realizou-se o desdobramento da interação por análise de regressão.

\section{RESULTADOS E DISCUSSÃO}

As fontes de fertilização e as doses utilizadas influenciaram de modo significativo $(P<0,05)$ a produção nos dois cortes realizados.

$\mathrm{Na}$ Tabela 3 são apresentados os resultados entre as medias de produção da Tifton $85 \mathrm{com}$ uso de dejeto in natura e biofertilizante nos dois cortes. Observa-se que com a aplicação de dejeto in natura a cv. Tifton 85 apresentou maior produção quando comparada com uso de biofertilizante, para ambos os cortes realizados. Estes resultados podem ser explicados pelo fato do dejeto in natura possuir maior concentração de nutrientes em comparação ao produto fermentado. Segundo Miranda et al. (2009), na obtenção do biofertilizante ocorre o processo de fermentação anaeróbica, resultando na redução de sólidos dissolvidos totais, bem como a quantidade de nutrientes.

TABELA 3 - Médias de produção (massa fresca e seca) da cv. Tifton 85 fertilizada com dejetos in natura e biofertilizante suíno em dois cortes

\begin{tabular}{|c|c|c|c|c|}
\hline \multirow{3}{*}{ Fontes } & \multicolumn{2}{|c|}{ Massa Fresca } & \multicolumn{2}{|c|}{ Massa seca } \\
\hline & $\mathrm{PC}$ & SC & $\mathrm{PC}$ & SC \\
\hline & \multicolumn{4}{|c|}{ - } \\
\hline Dejetos in natura & $10760,31 a$ & $11595,69 a$ & $4884,37 a$ & $4364,44 a$ \\
\hline Biofertilizante & $8347,44 b$ & $5827,94 b$ & $4005,62 b$ & $3200,44 b$ \\
\hline C.V. $(\%)$ & 13,1 & 13,4 & 13,7 & 12,7 \\
\hline DMS & 911,43 & 851,59 & 445,14 & 352,87 \\
\hline
\end{tabular}

*Médias não seguidas da mesma letra minúscula na coluna diferem entre si pelo teste Tukey ao nível de $5 \%$ de probabilidade. Em que: PC (primeiro corte); SC (segundo corte). 
ZENATTI , R. et al. Produtividade e fitodisponibilidade...

Observa-se na (Figura 1a) no primeiro corte realizado após os 28 dias do corte de nivelamento, que a produção de massa fresca apresentou um comportamento quadrático para o dejeto in natura e linear para o biofertilizante. A dose mais alta do dejeto in natura proporcionou a maior produção de gramínea que foi $16582 \mathrm{~kg} \mathrm{ha}^{-1}$, sendo que também a maior dose de biofertilizante atingiu a maior produção de $13134 \mathrm{~kg} \mathrm{ha}^{-1}$. Já para massa seca, ocorre um aumento linear para os dois fertilizantes (Figura 1b), com a dose de $600 \mathrm{~m}^{3}$ há ${ }^{-1}$ do dejeto in natura e do biofertilizante a produção foi de $7142 \mathrm{~kg}$ $\mathrm{ha}^{-1}, 5574 \mathrm{~kg} \mathrm{ha}^{-1}$, respectivamente.

No segundo corte, este realizado após os 56 dias do corte de nivelamento, a massa fresca au- mentou de forma quadrática para as duas fontes (Figura 1c). Para a fertilização com dejeto in natura a dose de $600 \mathrm{~m}^{3} \mathrm{ha}^{-1}$ resultou na maior produção da gramínea que foi de $17660 \mathrm{~kg} \mathrm{ha}^{-1}$, para o biofertilizante a produção teve um ponto de máximo da curva e foi atingido com a dose $513,43 \mathrm{~m}^{3} \mathrm{ha}^{-1}$ resultando na produção de $7991,98 \mathrm{~kg} \mathrm{ha}^{-1}$. A massa seca aumentou de forma quadrática para os dois fertilizantes (Figura 1d), o dejeto in natura proporcionou o máximo da produção com a maior dose que foi de $5757 \mathrm{~kg} \mathrm{ha}^{-1}$, para o biofertilizante a produção teve novamente um ponto de máximo que foi com dose de $392,80 \mathrm{~m}^{3} \mathrm{ha}^{-1}$ e resultou na produção de $3792 \mathrm{~kg} \mathrm{ha}^{-1}$.

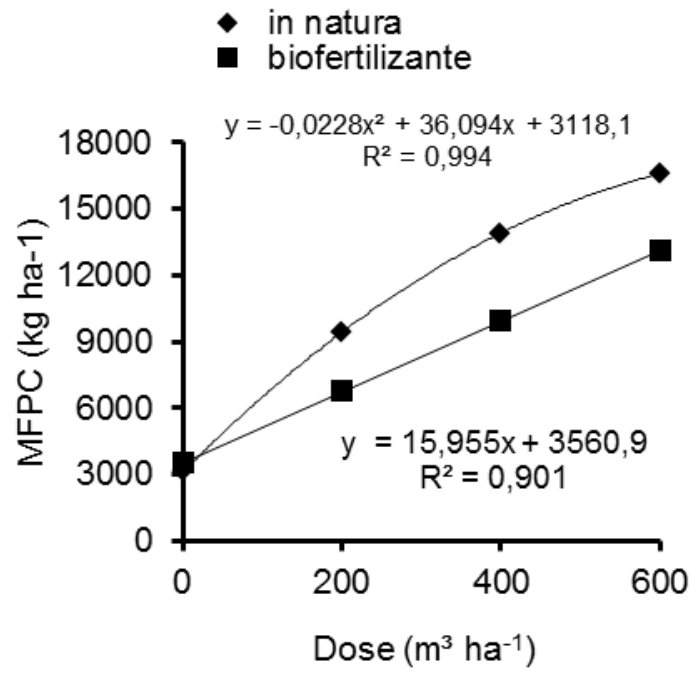

a)

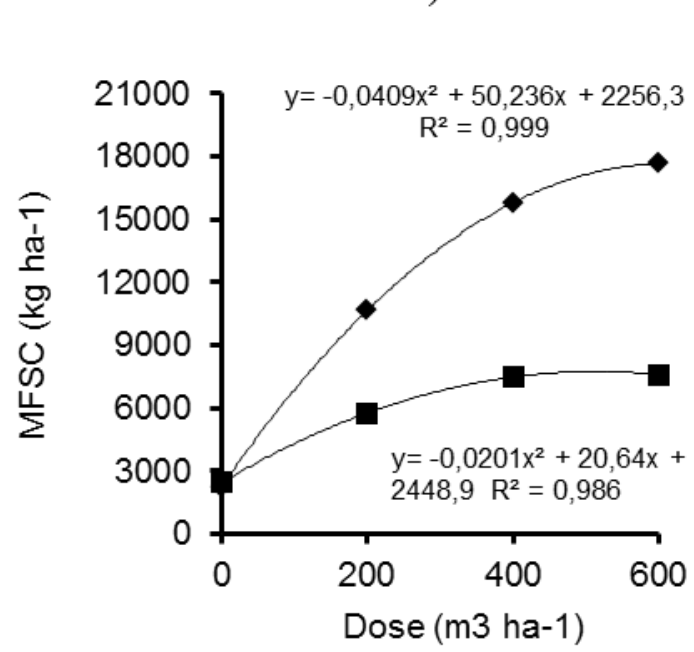

c)

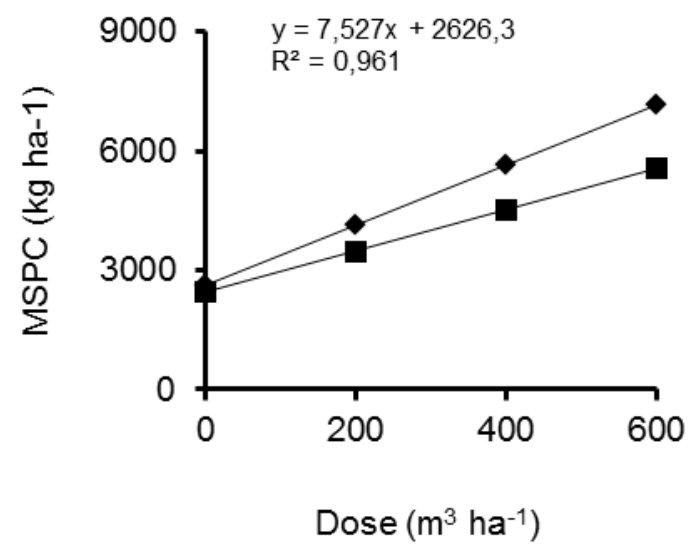

b)

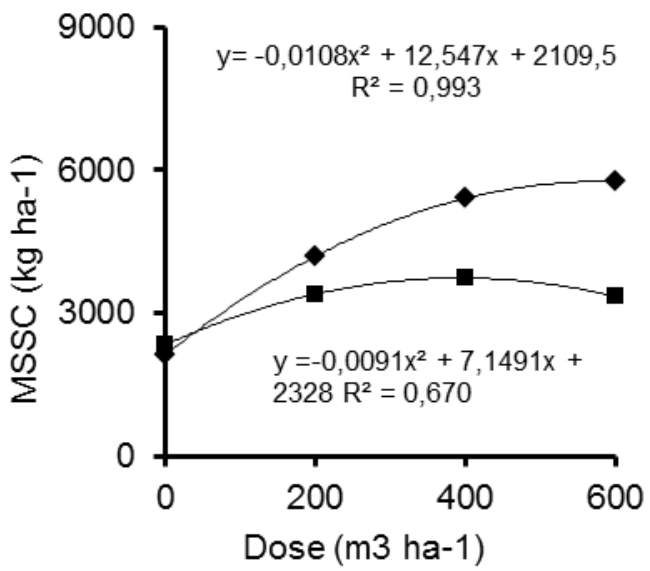

d)

FIGURA 1 - Produção de massa fresca primeiro corte (MFPC), segundo corte (MFSC), de massa seca no primeiro corte (MSPC) e segundo corte (MSSC) da cv. Tifton 85 fertilizada com diferentes doses de dejetos in natura e biofertilizante. 
ZENATTI , R. et al. Produtividade e fitodisponibilidade...

Como observado anteriormente na comparação de médias de produção, para as doses também houve superioridade do dejeto in natura quando comparado ao uso do dejeto estabilizado (biofertilizante) para produção da gramínea.

Os resultados da analise de variância para os teores de nutrientes no primeiro corte estão apresentados na Tabela 4.
Os fertilizantes orgânicos apresentaram diferença significativa $(P<0,05)$ no teor do elemento $\mathrm{Mn}$ no primeiro corte, sendo que as doses influenciaram significativamente nos teores $\mathrm{N}, \mathrm{P}, \mathrm{Cu}$ e Zn.

Os resultados da analise de variância para os teores de nutrientes no segundo corte da pastagem estão apresentados na Tabela 5.

TABELA 4 - Análise de variância para os teores de nutrientes na massa seca da cv. Tifton 85 no primeiro corte, realizado após 28 dias do corte de nivelamento

\begin{tabular}{|c|c|c|c|c|c|c|c|c|c|c|}
\hline \multirow{2}{*}{ F.V } & \multirow{2}{*}{ GL } & \multicolumn{9}{|c|}{ Quadrados médios } \\
\hline & & $\mathrm{N}$ & $\mathrm{P}$ & $\mathrm{K}$ & $\mathrm{Ca}$ & $\mathrm{Mg}$ & $\mathrm{Fe}$ & $\mathrm{Mn}$ & $\mathrm{Cu}$ & $\mathrm{Zn}$ \\
\hline $\begin{array}{c}\text { Fontes } \\
\text { (A) }\end{array}$ & 1 & $24.80^{N S}$ & $0.01^{\mathrm{NS}}$ & $0.53^{\mathrm{NS}}$ & $1.36^{N}$ & $0.26^{\mathrm{N}}$ & $10011^{\mathrm{NS}}$ & $101926^{*}$ & $12.50^{\mathrm{NS}}$ & $38.28^{\mathrm{NS}}$ \\
\hline $\begin{array}{l}\text { Doses } \\
\text { (B) }\end{array}$ & 3 & $\underset{*}{239.04}$ * & $1.08^{* *}$ & $4.55^{\mathrm{NS}}$ & $1,47_{S}^{N}$ & $0.15^{N}$ & $42801^{N S}$ & $24834^{\mathrm{NS}}$ & $37.02^{* *}$ & $124.86^{* *}$ \\
\hline$A \times B$ & 3 & $8.03^{\mathrm{NS}}$ & $0.02^{\mathrm{NS}}$ & $5.61^{\mathrm{NS}}$ & $2.91_{S}^{N}$ & $0,04^{N}$ & $49514^{N S}$ & $43056^{\mathrm{NS}}$ & $1.58^{\mathrm{NS}}$ & $125.41^{\mathrm{NS}}$ \\
\hline Resíduo & 24 & 12.13 & 0.12 & 2.34 & 2.92 & 0.39 & 18487 & 13076 & 4.62 & 20.32 \\
\hline C.V. & - & 18.69 & 14.26 & 10.78 & 19,90 & 10.36 & 15.75 & 10.29 & 16.40 & 15.23 \\
\hline M.G. & - & 18.63 & 1.78 & 14.21 & 8,64 & 2.39 & 187.93 & 267.25 & 6.37 & 29.59 \\
\hline
\end{tabular}

F.V (Fonte de Variação); GL (Graus de liberdade); ${ }^{* *}$ (significativo a 1\% pelo teste T de Student); ${ }^{\text {(significativo }}$ a $5 \%$ pelo teste $\mathrm{T}$ de student); NS (não significativo a $5 \%$ pelo teste $\mathrm{T}$ de student); C.V.(coeficiente de variação) M.G. (média geral, sendo os valores em $\mathrm{g} \mathrm{kg}^{-1}$ para o $\mathrm{N}, \mathrm{P}, \mathrm{K}, \mathrm{Ca}, \mathrm{Mg}$ e $\mathrm{mg} \mathrm{kg}^{-1}$ para o $\mathrm{Fe}, \mathrm{Mn}, \mathrm{Cu}, \mathrm{Zn}$ ).

TABELA 5 - Análise de variância para os teores de nutrientes na massa seca da cv. Tifton 85 no segundo corte,

\begin{tabular}{|c|c|c|c|c|c|c|c|c|c|c|}
\hline \multirow{2}{*}{ F.V } & \multirow{2}{*}{ GL } & \multicolumn{9}{|c|}{ Quadrados médios } \\
\hline & & $\mathrm{N}$ & $P$ & $\mathrm{~K}$ & $\mathrm{Ca}$ & $\mathrm{Mg}$ & $\mathrm{Fe}$ & $\mathrm{Mn}$ & $\mathrm{Cu}$ & $\mathrm{Zn}$ \\
\hline $\begin{array}{l}\text { Fontes } \\
\text { (A) }\end{array}$ & 1 & $20.33^{N S}$ & $1.79^{* *}$ & $5.28^{N S}$ & $0.76^{\mathrm{NS}}$ & $0.27^{\mathrm{NS}}$ & $104767^{* *}$ & $2592^{N S}$ & $392^{\star *}$ & $2.0^{\mathrm{NS}}$ \\
\hline $\begin{array}{l}\text { Doses } \\
\text { (B) }\end{array}$ & 3 & $191.6^{* *}$ & $8.24^{* *}$ & $79.5^{\star *}$ & $10.79^{\text {NS }}$ & $0.34^{\mathrm{NS}}$ & $42805^{* *}$ & $48098^{* *}$ & $259^{* *}$ & $186.87^{* *}$ \\
\hline$A \times B$ & 3 & $9.61^{\mathrm{NS}}$ & $0.52^{* *}$ & $4.36^{N S}$ & $5.41^{\mathrm{NS}}$ & $1.22^{\mathrm{NS}}$ & $60460^{* *}$ & $\underset{S}{21485^{N}}$ & $161^{* *}$ & $42.36^{\mathrm{NS}}$ \\
\hline $\begin{array}{c}\text { Resí- } \\
\text { duo }\end{array}$ & 24 & 280.6 & 0.12 & 3.72 & 3.65 & 0.39 & 5123 & 3878 & 28.0 & 50.91 \\
\hline C.V. & - & 18.74 & 14.22 & 12.77 & 21,23 & 26.81 & 15.30 & 19.91 & 16.30 & 9.47 \\
\hline M.G. & - & 18.24 & 2.45 & 15.10 & 9.00 & 2.33 & 174.53 & 312.75 & 11.25 & 33.81 \\
\hline
\end{tabular}

F.V (Fonte de Variação); GL (Graus de liberdade); ${ }^{* *}$ (significativo a 1\% pelo teste T de Student); (significativo a $5 \%$ pelo teste T de student); NS (não significativo a $5 \%$ pelo teste T de student); C.V.(coeficiente de variação) M.G. (média geral, sendo os valores em $\mathrm{g} \mathrm{kg}^{-1}$ para o N, P, K, Ca, Mg e mg kg-1 para o Fe, $\mathrm{Mn}, \mathrm{Cu}, \mathrm{Zn}$ ) . 
ZENATTI , R. et al. Produtividade e fitodisponibilidade...

Os fertilizantes orgânicos apresentaram diferença significativa $(P<0,05)$ nos teores dos elementos $\mathrm{P}$, Cu e Fe no segundo corte. As doses influenciaram significativamente nos teores $\mathrm{N}, \mathrm{P}, \mathrm{K}$, $\mathrm{Fe}, \mathrm{Mn}, \mathrm{Cu}$ e $\mathrm{Zn}$.

Os valores médios dos nutrientes em ambos cortes da gramínea fertilizada com dejetos in natura e biofertilizante estão apresentados na Tabela 6 .

Para os elementos $\mathrm{P}$ e $\mathrm{Mn}$ observa-se que o dejeto in natura proporcionou maior acúmulo no material vegetal da cv. Tifton 85, ocasionado, provavelmente, pelos maiores teores encontrados nesta fonte em comparação ao biofertilizante (Tabela 2). Já para os micronutrientes $\mathrm{Fe}$ e $\mathrm{Cu}$ o maior acúmulo ocorreu com o uso de biofertilizante, efeito que também pode ser explicado devido à maior concentração destes elementos nesta fonte.
O teor de $\mathrm{N}$ no tecido vegetal no primeiro corte e segundo corte aumentou linearmente em função da aplicação dos dois fertilizantes. De acordo Giacomini (2005), o N é o nutriente que normalmente possui maior concentração nos dejetos suínos, e também é o elemento mais utilizado e conseqüentemente mais absorvido pela gramínea, acarretando em um acúmulo proporcional ao aumento das doses de fertilizantes. O resultado do presente trabalho está de acordo com os citados por Fey (2006), que trabalhou com doses de dejetos no Tifton e também verificou que os teores $\mathrm{N}$ no tecido vegetal aumentaram significativamente de forma linear em função das doses.

Para o teor $\mathrm{P}$ em função das doses e o desdobramento entre os fertilizantes orgânicos está apresentada na Figura 3.

TABELA 6 - Resultados da comparação de médias para os teores de nutrientes da Tifton 85

\begin{tabular}{|c|c|c|c|c|}
\hline \multirow{3}{*}{ Fontes } & Teor de $\mathrm{Mn}$ & Teor de $\mathrm{P}$ & Teor de Fe & Teor de Cu \\
\hline & PC & SC & $\mathrm{SC}$ & SC \\
\hline & \multicolumn{4}{|c|}{ 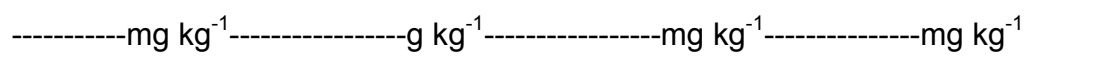 } \\
\hline Dejetos "in natura" & $323 a$ & $2,70 a$ & $117.31 b$ & $7,75 b$ \\
\hline Biofertilizante & $210 b$ & $2,22 b$ & $231.77 a$ & $14,75 a$ \\
\hline C.V. (\%) & 21,95 & 14,22 & 15,30 & 14,92 \\
\hline DMS & 83,44 & 0,25 & 52,23 & 3,92 \\
\hline
\end{tabular}

*Médias não seguidas da mesma letra minúscula na coluna diferem entre si pelo teste Tukey ao nível de $5 \%$ de probabilidade. Em que: PC (primeiro corte); SC (segundo corte).

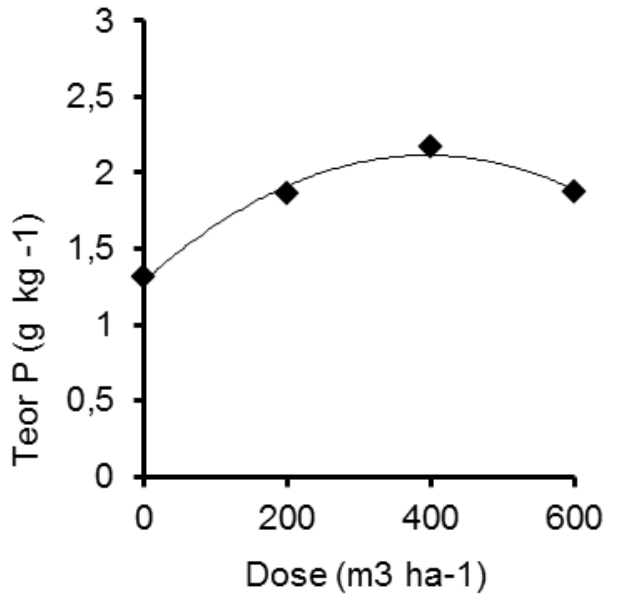

a) Primeiro Corte
- in natura $\quad$ abiofertilizante

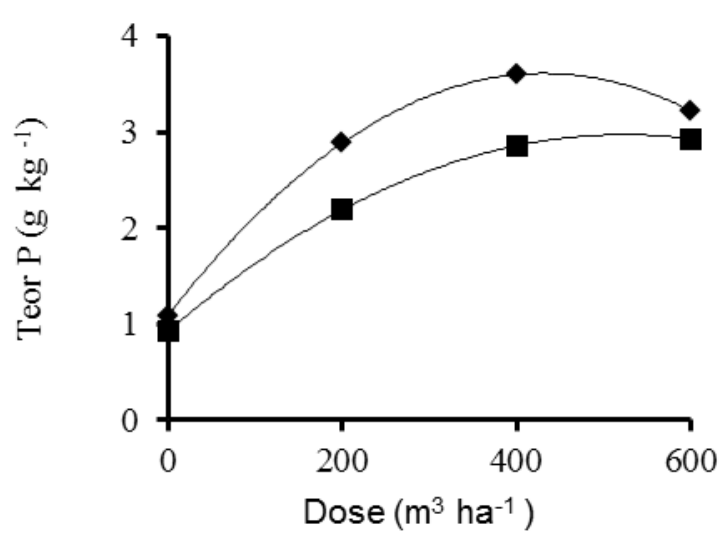

b) Segundo Corte

FIGURA 3 - Resultados dos teores de P em dois cortes da cv. Tifton fertilizada com dejeto in natura e biofertilizante. 
ZENATTI , R. et al. Produtividade e fitodisponibilidade...

Os teores de $\mathrm{P}$ no tecido vegetal do primeiro corte (Figura 3a) aumentaram significativamente em função das doses dos fertilizantes, a dose de 420 $\mathrm{m}^{3}$ ha $^{-1}$ proporcionou a maior disponibilidade do nutriente para gramínea. No segundo corte para as doses, na qual o dejeto in natura e biofertilizante apresentaram um ponto máximo de absorção do nutriente, sendo, respectivamente $417,85 \mathrm{~m}^{3} \mathrm{ha}^{-1} \mathrm{e}$ $557,1 \mathrm{~m}^{3} \mathrm{ha}^{-1}$, (Figura $3 \mathrm{~b}$ ). Os teores de $\mathrm{P}$ no tecido foliar estão de acordo com a faixa de nutrientes proposta por Werner et al. (1996), que varia de 1,5 a $3 \mathrm{~g} \mathrm{~kg}^{-1}$, sendo neste trabalho obtidas as médias de $1,78 \mathrm{~g} \mathrm{~kg}^{-1}$ e $2,45 \mathrm{~g} \mathrm{~kg}^{-1}$ no primeiro e segundo corte respectivamente.

$\mathrm{Na}$ Figura 4 é apresentada a análise de regressão para o teor $\mathrm{K}$ em função das doses no segundo corte.

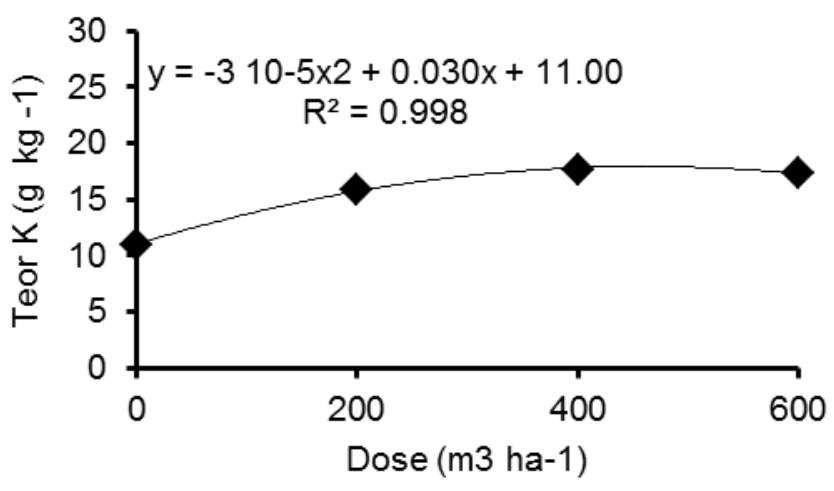

FIGURA 4 - Resultados dos teores de K no segundo corte da cv. Tifton fertilizada com doses crescentes de fertilizantes orgânicos.

$\mathrm{O}$ teor de $\mathrm{K}$ foi significativo em função das doses avaliadas no segundo corte apresentando um ponto de maior absorção com a dose de $500 \mathrm{~m}^{3}$ ha 1 . Estes resultados podem estar relacionados à disponibilidade deste elemento, que se apresenta na forma trocável $\left(\mathrm{K}^{+}\right)$nos fertilizantes utilizados e pela alta mobilidade em solução, o que facilita sua absorção pelas planta, (Neves et al, 2009) . O valor médio no teor de $\mathrm{K}$ para do primeiro corte $(14,21 \mathrm{~g}$ $\mathrm{kg}^{-1}$ ) ficou abaixo da faixa de nutrientes proposta por Werner et al, (1996) e no segundo corte $(15,10$ $\mathrm{g} \mathrm{kg}^{-1}$ ) o valor médio ficou dentro da faixa, sendo que a faixa ideal proposta varia de 15 a $30 \mathrm{~g} \mathrm{~kg}^{-1}$.

No primeiro corte o teor de Fe não foi significativo, conforme resultados da Tabela 5 . No segundo corte o teor de $\mathrm{Fe}$ foi significativo para as fontes e as doses, assim realizou-se o desdobramento da regressão quadrática. Para o biofertilizante o ponto máximo de absorção de $\mathrm{Fe}$ foi atingido com a dose de $272,80 \mathrm{~m}^{3} \mathrm{ha}^{-1}$. O aumento na disponibilidade do Fe em biofertilizante também foi constatado por Sediyama et al, (2007) e enfatizam que isso é consequência mineralização da matéria orgânica presente neste fertilizante. O teor médio de $\mathrm{Fe}$ no tecido vegetal ficou acima da faixa de nutrientes proposta por Werner et al, (1996) que varia de 4 a $20 \mathrm{mg} \mathrm{kg}^{-1}$ e as médias obtidas neste experimento foram de $187,93 \mathrm{mg} \mathrm{kg}^{-1}$ e $174,53 \mathrm{mg} \mathrm{kg}^{-1}$ no primeiro e segundo corte respectivamente.

$\mathrm{O}$ teor de $\mathrm{Mn}$ foi significativo para as doses no segundo corte e caiu de forma linear com uso dos dois fertilizantes (Figura 5). Segundo Reinheimer et al. (1998) a disponibilidade de Mn é reduzi- da com elevação $\mathrm{pH}$ do solo, principalmente pela complexação com ânions gerados pela calagem no solo, pratica realizada neste trabalho. Outro fator para sua redução seria pelo alto teor da matéria orgânica no solo que forma complexos estáveis com Mn (Lopes, 1999). As concentrações de Mn no tecido foliar com o uso das duas fontes de fertilização estão de acima da faixa proposta Werner et al (1996) que varia de 15 a $70 \mathrm{mg} \mathrm{kg}^{-1}$.

Com relação ao teor de $\mathrm{Cu}$ no tecido foliar observa-se que o aumento das doses acarreta em aumento de forma linear para as duas fontes no primeiro corte (Figura 6a). No segundo corte houve interação entre as fontes e doses o teor se elevou de forma linear, sendo que neste corte apresentou maior teor que no primeiro corte (Figura 6b). O aumento no teor do cobre pode ser explicado pela adição deste nutriente via fertilizante, sendo que resultados estão de acordo com Burns et al. (1990). Os teores de $\mathrm{Cu}$ no tecido vegetal ficaram abaixo da faixa de nutrientes proposta por Werner et al. (1996) que varia de 50 a $200 \mathrm{mg} \mathrm{kg}^{-1}$ e as médias obtidas neste para este trabalho foram $11,25 \mathrm{mg} \mathrm{kg}$ ${ }^{1}$ e $16,40 \mathrm{mg} \mathrm{kg}^{-1}$.

$\mathrm{O}$ aumento significativo para o teor de $\mathrm{Zn}$ no tecido vegetal está relacionado ao teor deste elemento na caracterização do solo, e ainda, na composição das fontes utilizadas, que de acordo com L'Herroux et al. (1997) e Grabert et al. (2005) o Zn é um dos principais metais pesados encontrados nos dejetos de suínos, usado em grande escala nas dietas dos animais em crescimento. Os teores de $\mathrm{Zn}$ no tecido vegetal ficaram dentro da faixa de nu- 
ZENATTI , R. et al. Produtividade e fitodisponibilidade...

trientes proposta por Werner et al. (1996) que varia de 20 a $300 \mathrm{mg} \mathrm{kg}^{-1}$ e as médias neste trabalho foram de $29,59 \mathrm{mg} \mathrm{kg}^{-1}$ e $33.81 \mathrm{mg} \mathrm{kg}^{-1}$ no primeiro e segundo corte respectivamente.

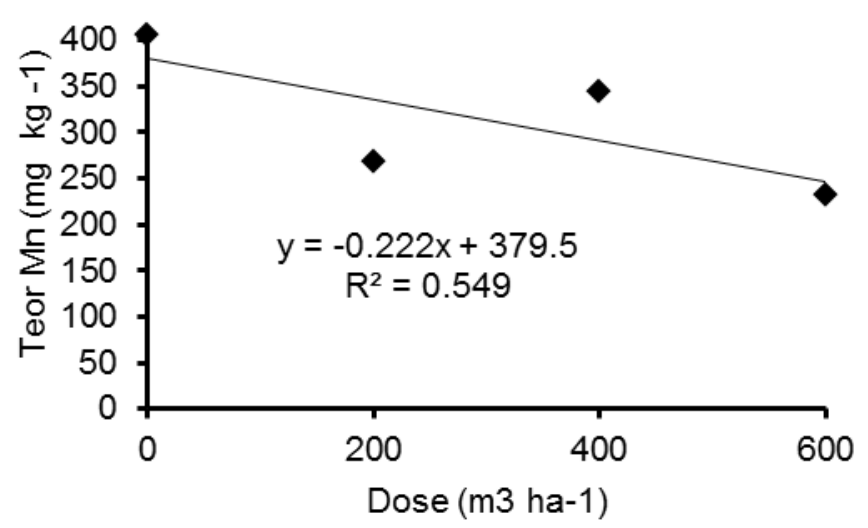

FIGURA 5 - Resultados dos teores de Mn em do segundo corte da cv. Tifton fertilizada com doses crescentes de fertilizantes orgânicos.

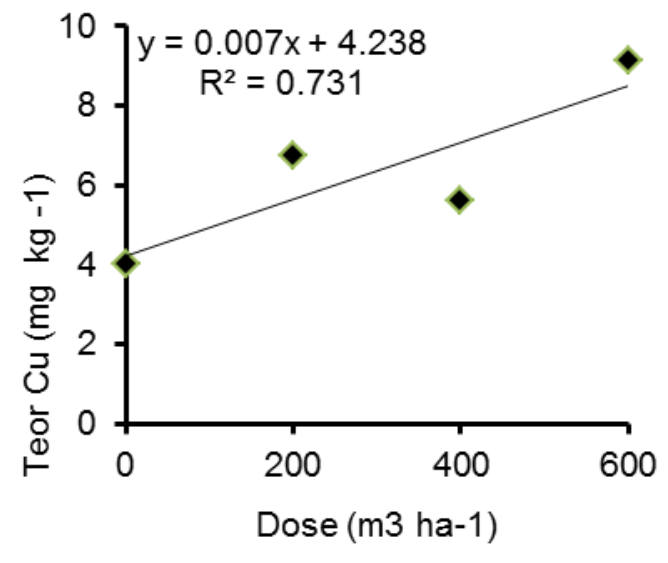

a) Primeiro Corte

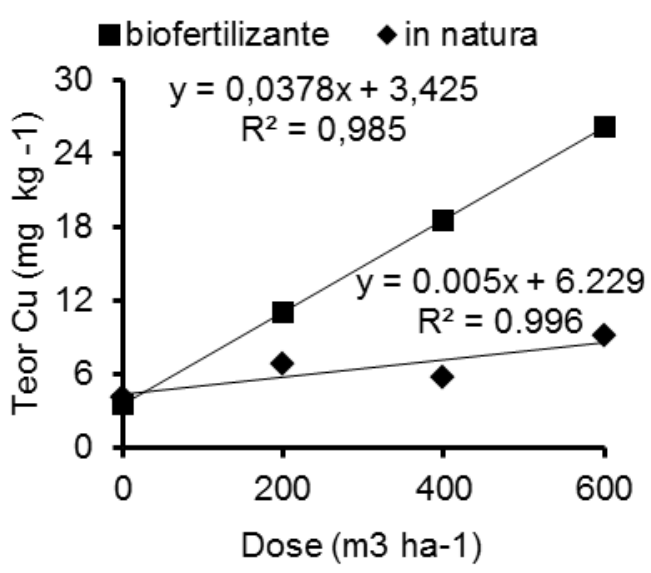

b) Segundo Corte

FIGURA 7 - Resultados dos teores de Cu em dois cortes da cv. Tifton fertilizada com dejetos in natura e biofertilizante

\section{CONCLUSÕES}

Com a aplicação de dejetos in natura a cv. Tifton 85 apresentou maior produção quando comparada com uso de biofertilizantes. O uso de dejetos in natura proporcionou um aumento significativo de produção de Tifton 85 até a maior dose aplicada.
Os nutrientes $\mathrm{N}, \mathrm{P}$ e $\mathrm{K}$ e os micronutrientes $\mathrm{Fe}, \mathrm{Cu}$ e $\mathrm{Zn}$ tiveram seu teor elevado em função das doses aplicadas e das fontes utilizadas, sendo que os teores de $\mathrm{Mn}$ foram reduzidos em função das doses aplicadas. 
ZENATTI , R. et al. Produtividade e fitodisponibilidade...

\section{REFERENCIAS}

1. ABREU, C. A.; LOPES, A. S.; SANTOS, G. Micronutrientes. In: NOVAIS, R. F. et al. (Ed.). Fertilidade do solo. Viçosa, MG: Sociedade Brasileira de Ciência do Solo, 2007. p. 645-736.

2. ANDRADE, C.S.; FONSECA, D.M.; GOMIDE, J.A.; ALVAREZ., V.H.; MARTINS, C.E. SOUZA, D.P.H. Produtividade e valor nutritivo do capim-elefante cv. napier sob doses crescentes de nitrogênio e potássio. Revista Brasileira de Zootecnia., 29:1589-1595, 2000.

3. ASSOCIATION OF OFFICIAL ANALYTICAL CHEMISTS - International [AOAC]. 2005. Official methods of analysis 18ed. AOAC, Gaithersburg, MD, USA.

4. BURNS, J. C., L. D. KING AND P. W. WESTERMAN. Long-term swine lagoon effluent applications on Coastal Bermuda grass. I. Yield, quality, and element removal. Journal of Environmental Quality. 19:749-756, 1990.

5. DRUMOND, L.C.D., ZANIN,. J.R., AGUIAR, A.P.A.. RODRIGUES, G., FERNANDES , A.L.T. Produção de matéria seca em pastagem de Tifton 85 irrigada, com diferentes doses de dejeto líquido de suíno. Engenharia Agrícola Vol. 26 Jabotical. 2006.

6. DURIGON, R.; CERETTA, C.A.; BASSO, C.J.;BARCELLOS, L.A.R. PAVINATO, P.S. Produção de forragem em pastagem natural com o uso de esterco líquido de suínos. Revista Brasileira de Ciência do Solo. Viçosa, v.26, n.4, p.983-992, 2002.

7. EMPRESA BRASILEIRA DE PESQUISA AGROPECUÁRIA [Embrapa]. 2006. Sistema brasileiro de classificação de solos. 2ed. Embrapa Solos, Rio de Janeiro, RJ, Brasil.

8. FERREIRA. D.F. 2003. SISVAR: Sistemas de análises estatísticas. UFLA, Lavras, MG, Brasil.

9. FEY, R. Teores de Nutrientes no solo, produção de fitomassa e qualidade da pastagem de Tifton 85 , produzida em área submetida à aplicação de dejetos de suínos. 2006 51p. Tese (Doutorado em Agronomia). Universidade Estadual de Londrina, Londrina.

10. GIACOMINI, S.J. Avaliação e modelização da dinâmica de carbono e nitrogênio com o uso de dejetos de suínos. 2005 240p. Tese (Doutorado em Ciência do Solo) .Santa Maria, Universidade Federal de Santa Maria.

11. GONÇALVES JR., A.C.; LINDINO C. A.; ROSA F. M.; BARICATTI R.; GOMES, G.F. Remoção de metais pesados tóxicos cádmio, chumbo e cromo em biofertilizante suíno utilizando macrófita aquática (Eichornia crassipes) como bioindicador. Acta Scientiarum 30: 9-14, 2008.

12. GRABERT, I. ET AL. Accumulation of copper and zinc in danish agricultural soils in intensive pig production areas. Danish Journal of Geography. vol. 105, n. 2, p. 15-22, 2005.

13. Instituto Agronômico do Paraná- [lapar]- Sugestão de recomendação de fertilização para culturas de interesse econômico do Paraná. Circular técnica 128. Londrina 2003, 30p.

14. L'HERROUX, L. et al. Behaviour of metals following intensive pig slurry applications to a natural field treatment process in Brittany (France). Environmental Pollution, v. 97, n. 1, p. 119-130, 1997

15. LOPES, A.S. Micronutrientes: filosofias de aplicação e eficiência agronômica. São Paulo: ANDA, Boletim técnico $\mathrm{n}^{\circ} 8,1999.58 \mathrm{p}$.

16. MALAVOLTA, E.; VITTI, G.C.; OLIVEIRA, S.A. 1997. Avaliação do estado nutricional das plantas: princípios e aplicações. 2ed. Potafos, Piracicaba, SP, Brasil.

17. MIRANDA, C. R. D; SANTOS FILHO, J. I.; A situação dos Dejetos Suínos na Região da AMAUC - SC, X Congresso Brasileiro de Veterinários Especialistas em Suínos, 26 a 29 de outubro de 1999, Resumos, Belo Horizonte, MG.

18. MIRANDA, A. P.; LUCAS JUNIOR, J.\& THOMAZ, M. C. 2009. Redução de sólidos e produção de biogás em biodigestores abastecidos com dejetos de suínos alimentados com dietasformuladas com milho ou sorgo. In: Simpósio Internacional sobre Gerenciamento de Resíduos de Animais Geração de Energia a partir de Resíduos Animais, I. Resumos, Florianópolis.

19. NEVES, L. S., ERNANI, P. R., SIMONETE, M. A., Mobilidade de potássio em solos decorrente da adição de doses de cloreto de potássio. Revista Brasileira Ciência do Solo, 33:25-32, 2009.

20. PAVAN, M.A.; BLOCH, M.F.; ZEMPULSKI, H.C.; MIYAZAWA, M.; ZOCOLER, D.C. Manual de análises químicas de solo e controle de qualidade. IAPAR, 1992. Londrina, PR, Brasil.

21. QUEIROZ, F. M.; MATTOS, A. F.; PEREIRA, O. G., OLIVEIRA, R. A. Características químicas de solo submetido ao tratamento com esterco líquido de suínos cultivado com gramíneas forrageiras. Ciência Rural, v. 34, n. 5, p. 14871492, 2004.

22. REINHEIMER, D.S.; KAMINSKI, J; LUPATINI, G.C. \& SANTOS, E.J.S. Modificações em atributos químicos de solos arenoso sob sistema plantio direto. Revista Brasileira Ciência do Solo, 22:713-722, 1998.

23. Santos, N., L. Produção e valor nutritivo dos capins Tifton 85, Tanzânia e Marandu sob irrigação suplementar. 2006 52p. Dissertação Mestrado. UESB (Programa de pós Graduação em Zootecnia Itapetinga-BA

24. SARMIENTO, P., NASCIMENTO, R.C., MARTINS, A.T., DA CRUZ, M.C.P., FERREIRA, M. E. Nutrientes limitantes ao desenvolvimento do capim-Tifton 85 em Argisssolo Vermelho Amarelo. Boletim da Indústria Animal, v. 63, p. 1118,2006

25. SEDIYAMA, M. A. N.; GARCIA, N. C. P.; VIDIGA, S. M.; MATOS, A. T. Nutrientes em compostos orgânicos de resíduos vegetais e dejetos de suínos. Scientia Agricola, v. 57, n. 1, p.185-189, 2007.

26. VILELA, D., ALVIM, M.J.; CAMPOS, O.F. Produção de leite de vacas Holandesas em confinamento ou em pastagem de Coastcross. Revista da Sociedade Brasileira de Zootecnia, v. 25, n. 6, p. 1228-1244, 1996.

27. WELZ, B.; SPERLING, M. 1999. Atomic Absorption Spectrometry. 2ed. Wiley-VCH, Weinheim, BW, Alemanha.

28. WERNER, J. C.; PAULINO, V. T.; CANTARELLA, H., ANDRADE, N. O; QUAGGIO, J. A. Forrageiras. In: Recomendações de fertilização e calagem para o Estado de São Paulo. 2. ed. Campinas: Fundação IAC, 1996. p. 263-274 (IAC. Boletim Técnico, 100). 